\title{
sciendo
}

RESEARCH PAPERS FACULTY OF MATERIALS

SCIENCE AND TECHNOLOGY IN TRNAVA

SLOVAK UNIVERSITY OF TECHNOLOGY

IN BRATISLAVA

2019, Volume 27, Number 45

DOI 10.2478/rput-2019-0027

\section{PROBLEMS OF A UTOPIA POINT SETTING IN TRANSFORMATION OF INDIVIDUAL OBJECTIVE FUNCTIONS IN MULTI-OBJECTIVE OPTIMIZATION}

\author{
Pavel VAŽAN ${ }^{1}$, Zuzana ČERVEŇANSKÁ ${ }^{1}$, Janette KOTIANOVÁ ${ }^{1}$, \\ Jiří HOLÍK ${ }^{2}$ \\ ${ }^{1}$ Slovak University OF TECHNOLOGY IN BRATISLAVA \\ FACULTY OF MATERIALS SCIENCE AND TECHNOLOGY IN TRNAVA \\ INSTITUTE OF APPLIED INFORMATICS, AUTOMATION AND MECHATRONICS \\ ULICA JÁNA BOTTU 2781/25, 91724 TRNAVA SLOVAK REPUBLIC \\ e-mail:pavel.vazan@stuba.sk,zuzana.cervenanska@stuba.sk,janette.kotianova@stuba.sk \\ ${ }^{2}$ DyNAMic Future, S.R.O., OBČANSKÁ 1117/23, 71000 OSTRAVA, CZECH REPUBLIC \\ e-mail: jiri.holik@dynamicfuture.cz \\ Received 28 August 2019, accepted 1 October 2019, published 29 November 2019
}

\begin{abstract}
In an optimal processes control, where the considered goals are in general observed as concurrently conflicted, a multi-objective approach fits the best. Commonly used scalarization techniques in multi-objective optimization need a transformation of the individual singleobjective functions involved into a scalar multi-criteria objective function. There are many parameters which can influence the optimization results solutions, including an unreachable utopia point value. In this study, the authors compare the multi-objective problem solutions found via two ways of the individual objectives transformation with the respect to setting the utopia point. The methods are used in the area of production control in a case study for a batch production system. To find the solutions, The Weighted Sum Method with a priori articulated preferences under specific constraints as the scalar multi-objective optimization method is applied in simulation optimization.
\end{abstract}

Key words

Multi-Objective Optimization, Simulation Optimization, Weighted Sum Method, Utopia Point

\section{INTRODUCTION}

Nowadays, a continual challenge for an automation of the control complex processes is to find more effective methods for prediction and optimization of their vital parameters. Especially, the control of the systems where the goals are typically contradictory necessarily demands a multi-objective optimization approach. The modelling and simulation techniques belong to the helpful tools thanks to their role in processes understanding and design 
optimization. The discrete-event simulation in a connection to the heuristic multi-objective optimization techniques can be useful in providing a holistic view of the optimization problem, offering thus better and preferable solutions.

Up to now, the possibilities of using the simulation optimization linking with the multiobjective optimization methods in real practice have not been fully investigated. In the production control area, which is the application domain of this study, a review of an application of simulation optimization in the manufacturing system design and operations including the multi-objective optimization is provided by Negahban and Smith [1]. The contribution of Hunter et al. [2] provides the theory, methods and algorithms for the multi-objective simulation optimization (MOSO) while focusing on finding the entire efficient set of solutions for MOSO problem on finite sets, with integer-ordered and continuous decision variables too. Also, Amaran et al. [3] offer a comprehensive overview of methods and applications of simulation optimization. Commonly used scalar multi-objective optimization is of a parametric nature; influence of the parameters' settings on the resulting solutions is still not completely clear.

\section{SCALAR MULTI-OBJECTIVE OPTIMIZATION}

Solving a general multi-objective optimization (MOO) problem means a route of a systematic and simultaneous optimization of a set of $k$ individual objective functions [4]. It can be defined in the following form (1):

$$
\begin{aligned}
\underset{\boldsymbol{x} \in D}{\operatorname{Minimize}} & \boldsymbol{F}(\boldsymbol{x})=\left[F_{1}(\boldsymbol{x}), F_{2}(\boldsymbol{x}), \ldots, F_{k}(\boldsymbol{x})\right]^{T} ; k \geq 2 \\
\text { subject to } & g_{s}(\boldsymbol{x}) \leq 0, s=1,2, \ldots, m ; m \geq 0, \quad h_{l}(\boldsymbol{x})=0, l=1,2, \ldots, r ; r \geq 0
\end{aligned}
$$

$m$ and $r$ are the number of inequality and equality constraints. The vector $x \in D, D \subseteq \mathrm{E}^{n}$ is the vector of $n$ design variables under constraints given by $\boldsymbol{x}_{\mathrm{L}} \leq \boldsymbol{x} \leq \mathbf{x}_{\mathrm{U}}$. The set $D$ is the feasible region in the design space. The vector $\boldsymbol{F}(\boldsymbol{x}) \in \mathrm{E}^{k}$ is the vector of objective functions $F_{i}(\mathbf{x})$ : $\mathrm{E}^{n} \rightarrow \mathrm{E}^{1}$ in the feasible objective space $\mathrm{Z}, \mathrm{Z} \subset \mathrm{E}^{k}$. If the objectives $F_{i}(\mathbf{x})$ are contradictory, it does not exist one best solution as a result of the multi-objective optimization. The result is the set of the optimal tradeoffs called Pareto optimum solutions [5]. The Pareto optimum solutions set is defined as a set of such feasible solutions in the objective function space, where there exist no other feasible solutions for each of them that will yield an improvement in one objective without causing degradation in at least one other objective [5].

\section{Scalarization, Weighted Sum Method and a transformation of individual objective functions}

In a simulation model-driven multi-objective optimization, a scalar form of an objective function is frequently used. The scalarization converts the MOO problem into a parameterized single-objective problem. Under appropriate regularity conditions, solving it results in an efficient point. Varying the scalarization parameters and solving each resulting problem creates a set of efficient points and a characterization of the Pareto set in the objective function space [2]. There are a lot types of scalarizing functions, the most preferred is the Weighted Sum Method (WSM). The linear weighted sum creates a scalar single-objective function $U$ with the weighted objectives in the form of their linear combination [4]. In this case, the scalarization parameters are the weights $w_{i}$ for each individual objective $F_{i}$ defined by the user preferences for a priori use. Regarding to the transformation of the objectives $F_{i}$ to gain a dimensionless, the $U$ function will be defined as follows (2):

$$
U=\sum_{i=1}^{k} w_{i} . F_{i}^{\text {transform }}, w_{i}>0 \forall i, \sum_{i=1}^{k} w_{i}=1
$$


According to [4], if we consider a convex Pareto front, a convex design space and a positive weights, minimizing $U$ function generates the Pareto optimal solution. The robust transformation $F_{i}^{\text {transform }}$ of all objectives $F_{i}$ employs transformation the upper-lower-bound normalization approach adapted from [6]. In the case of minimization, the transformation of individual objective $F_{i}$ in the form (3) is constructed on the relation to a component of a utopia point $F_{i}^{U}$ and a nadir point $F_{i}^{N}$.

$$
F_{i}^{\text {transform }}=\frac{F_{i}-F_{i}^{U}}{F_{i}^{N}-F_{i}^{U}} .
$$

The following definitions including expressions (4) and (5) explain the meaning of the utopia and nadir points.

Definition 1. A point is $\boldsymbol{F}^{\boldsymbol{U}}, \boldsymbol{F}^{\boldsymbol{U}} \in Z$ is a utopia point iff for each $i=1,2,3, \ldots, k$

$$
F_{i}^{U}=\underset{x}{\operatorname{minimum}}\left\{F_{i}(\boldsymbol{x}) \mid \boldsymbol{x} \in D\right\} .
$$

Definition 2. A point $\boldsymbol{F}^{N}, \boldsymbol{F}^{N} \in Z$ is a nadir point iff for each $i=1,2,3, \ldots, k$

$$
F_{i}^{N}=\max _{1 \leq j \leq k} F_{i}\left(x_{j}^{*}\right)
$$

The situation is depicted at Figure 1 . The point $x_{j}^{*}$ minimizes the $j$-th objective function. $x_{j}^{*}$ is a vertex of the Pareto optimal set in the design space and $\boldsymbol{F}\left(\boldsymbol{x}_{j}^{*}\right)$ is a vertex of the Pareto optimal set in the objective space. Such a maximum is called the Pareto maximum [6]. It approximates the nadir vector, which has components defined by the upper bounds of the Pareto optimal set in the objective space, but is difficult to determine exactly. Like an alternative, $F_{i}{ }^{N}$ may also be determined using the absolute maximum of $F_{i}(\boldsymbol{x})$. According to Marler [4], $F_{i}^{U}$ and $F_{i}^{N}$ values are possible to approximate by an engineering intuition. Authors in [7, 8] applied the substitution by the optimal values obtained as the solutions of the single-objective optimization.

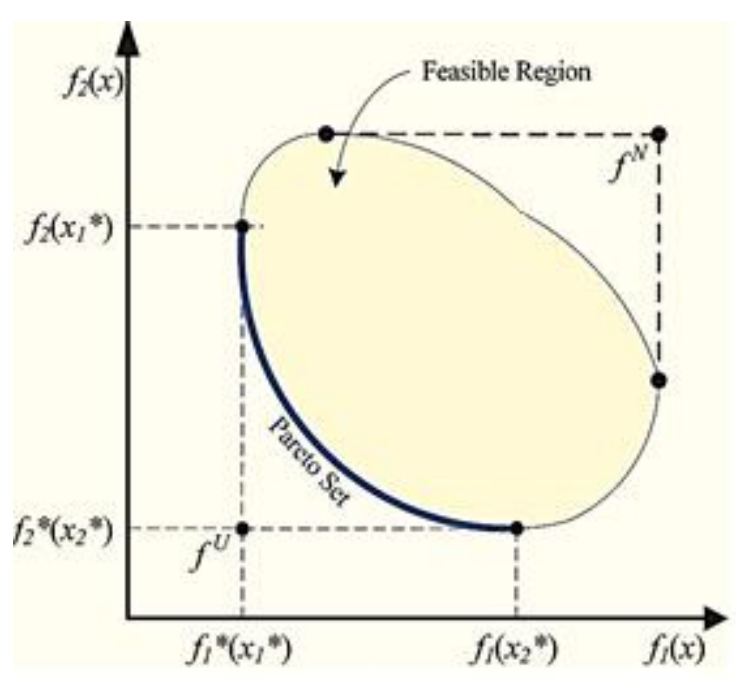

Figure 1 Pareto front and utopia and nadir points for two-objective optimization problem. Both objectives are minimized. Adapted from [9]. 


\section{THE OPTIMIZATION EXPERIMENTS METHODOLOGY}

The MOO problem chosen for the exploring an influence of the utopia point setting touches the batch production systems control. In the experimental study using an event-driven simulation model of the production system, we have focused on comparing two approaches to the individual objective functions transformation (3). Both types of experiments with the minimizing the scalar multi-objective function including the transformed individual functions were aimed to observe the influence of setting utopia point to vector of production goals under the control in the objective space.

\section{Transformation of production goals}

Applied transformations are characterized by an adaption of the expression (3) to apply the simulation optimization related to the limits of the production system and the best production goals $F_{i}^{U}$ which are unreachable in practice. When taking it into account, the expression (3) takes the form (6):

$$
F_{i}^{\text {transform }}=\frac{F_{i}-F_{i}^{U}}{F_{i}^{\text {optim }}-F_{i}^{U}} .
$$

In our study, the utopia point forms an unreachable solution as four-dimensional vector $\boldsymbol{F}^{\boldsymbol{U}}$ in the objective space Z. It represented four selected production goals $F_{i}^{U}$ for the studied production system as it is defined in (7):

$\boldsymbol{F}^{\boldsymbol{U}}=$ (flow time, costs per part unit, machines utilization, number of products).

Two applied transformations of individual functions were as follows:

- Transformation 1. The first experiment was based on the scalar objective function contained the expression (6) where $F_{i}^{U}$ was taken as the best result of single-objective optimization and $F_{i}^{\text {optim }}$ was substituted by production limits of the system determined by preliminary experiments on simulation model which were the same as constraints in single-objective optimization. This transformation lead to the single solution for specified production limits mentioned above.

- Transformation 2. The second group of experiments differed in that the meaning of values $F_{i}^{U}$ and $F_{i}^{\text {optim }}$ in the transformation (6) was interpreted differently. In this case, the value $F_{i}^{U}$ was a (unattainable) component of the utopia point vector $\boldsymbol{F}^{\boldsymbol{U}}$ for each of the individual objectives regardless to the real capability of the production system. It represented a vector that dominates the entire set of potential solutions $\boldsymbol{F}(\boldsymbol{x})$ in the objective space but it lies outside the feasible space $Z$. The best utopian values converge to zero for both the flow time and costs per part unit and, in contrast, the utopian value of the number of products converges to infinity. One of three coordinates of the utopia point vector $F_{i}^{U}$ subjected to a consequent change meanwhile others were fixed as follows: flow time $=1$ minute, costs per part unit $=1$ euro, machine utilization $=100 \%$ and a number of products $=2000$ pcs. A range of values for a utopia point in the transformation is defined in Table 1 . The value $F_{i}^{\text {optim }}$ was here as the minimum or maximum of constrained single-objective function according to the optimization goal regardless other objective. It was yielded by the simulation optimization. The vector $\boldsymbol{F}^{\text {optim }}$ was composed of the optimum values for each objective function $F_{i}^{\text {optim }}$. 


\begin{tabular}{|l|c|}
\hline \multicolumn{2}{|l|}{$\begin{array}{l}\text { Table } 1 \text { Range of components for a utopia point vector in the transformation } 2 \text { of objective } \\
\text { functions } F_{i}\end{array}$} \\
\hline & Range of utopian value $\boldsymbol{F}_{\boldsymbol{i}}^{\boldsymbol{U}}$ \\
\hline Flow time [min.] & $0.01-250$ \\
\hline Costs per part unit [€] & $0.001-10$ \\
\hline Machines utilization [\%] & 100 \\
\hline Number of products [pcs] & $300-10000$ \\
\hline
\end{tabular}

The production goals $F_{i}$ in expression (6) reflecting the system structure and its parameters were monitored by the discrete-event simulation. They entered into the scalar objective function $U(2)$ in the optimization model in studied MOO problem.

\section{The Optimization Model of the Production System}

The model production system chosen for the study was a job shop category of batch production which produced two different products $\mathrm{P} 1$ and $\mathrm{P} 2$. It comprised eight workstations with the automated parallel working machines and three operators. Each of these products was finishing individually at the end of producing. Six vehicles on the tracks delivered processing products among workstations. The model was fully deterministic. We fixed all settings of inner system parameters such as setup times and operation times for all machines within all performance of the optimization experiments. Costs for operators, machine setups and storage were fixed as well. The discrete-event simulation model of the system was created in the simulation software Witness.

A change of the input parameters as the lot size and an arrival time into the system for products $\mathrm{P} 1$ and $\mathrm{P} 2$ was representing a varying the system loading. A design space existed as a discrete four-dimensional domain, bounded by lower and upper limits for the lot size in the range from 2 to 10 pieces and input arrival time in the range from 5 to 50 minutes, the same for both of products.

This model system - with added the same constraints but different objective function served for all either single or multi-objective optimization experiments. The scalar multiobjective function $U$ was created in sense of expression (2) using two types of transformations (6) consequently. Weight values $w_{i}=0.25$ were the same for all transformed individual functions $F_{i}^{\text {transform }}$. All applied constraints can be seen in Table 2 .

Table 2 Constraints for a single and multi-objective optimization

\begin{tabular}{|l|c|}
\hline & Constraint value \\
\hline Max. flow time [min.] & 550 \\
\hline Max. costs per part unit [€] & 16 \\
\hline Min. machines utilization [\%] & 60 \\
\hline Min. number of products [pcs] & 230 \\
\hline
\end{tabular}

The scalar multi-objective optimization for both forms of transformation was carrying out within an Advanced mode of module Experimenter - a software Witness component. The heuristic methods like Thermoadaptive Simulated Annealing and Random Solutions were combining with a brute force algorithm All Combinations to obtain the global minimum of the scalar function $U$ constrained by values shown in Table 2 . One replication for one scenario with run length 1440 minutes and warm-up period 100 minutes was performed. 


\section{RESULTS OF OPTIMIZATION EXPERIMENTS}

Firstly, preliminary experiments showed the limits of the studied production system. Then the single-objective optimization for all four production goals consequently was performed. It found the best values $F_{i}^{\min }, F_{i}^{\max }$ resp., for each individual production goal irrespective of three other ones for the purpose of a normalization of the individual production goals entering to the scalar multi-objective function $U$. The obtained results are demonstrated in Table 3.

\begin{tabular}{|l|c|}
\hline Table 3 Experimental results of single-objective optimization \\
\hline Flow time [min.] & $\boldsymbol{F}_{\boldsymbol{i}}^{\text {optim }}$ \\
\hline Costs per part unit [€] & 296.309 \\
\hline Machines utilization [\%] & 10.209 \\
\hline Number of products [pcs] & 83.895 \\
\hline
\end{tabular}

Secondly, the results of single-objective optimization were used for transforming the individual functions of production goals $F_{i}$ regarding expression (6) and a global multiobjective optimization with different type of transformation in scalar objective function $U$ was carrying out.

The effect the utopia point setting (a change in the average flow time, costs per part unit and the number of product component) applying transformation 2 on four production goals is depicted in Figure 2. The resume of experimental results in the form of the graphical comparison of the effect of applying two different transformations on observed production goals with corresponding design vectors is shown in Figure 3.

a

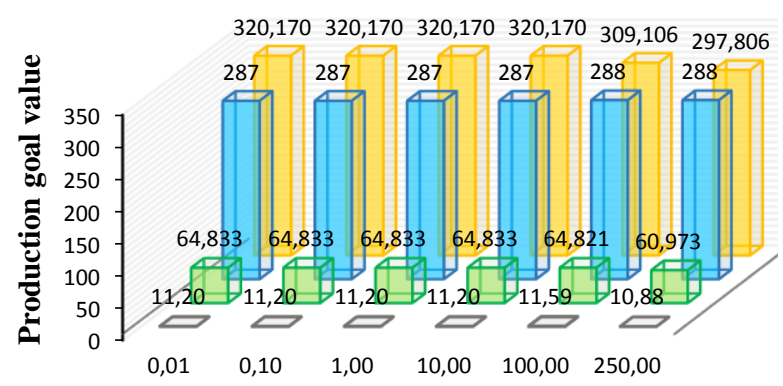

Flow time in the utopia point [min]

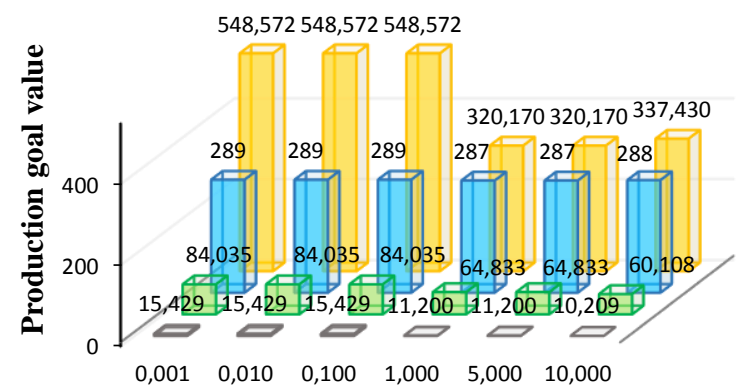

Costs per part unit in the utopia point $[€]$

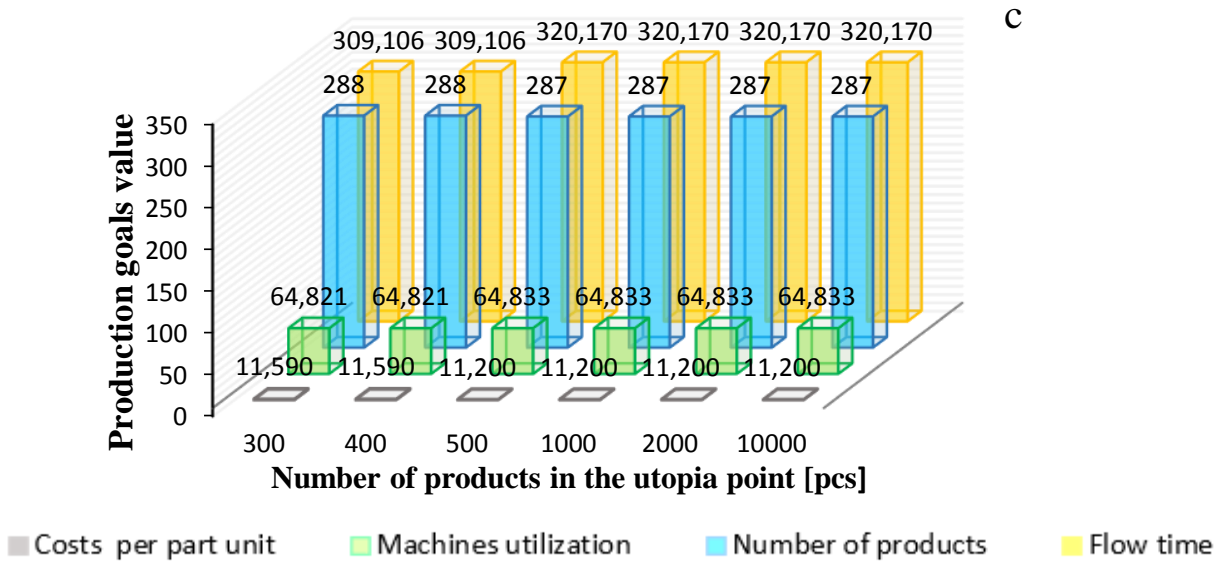

Figure 2 The effect of the change of flow time (a), costs per part unit (b) and the number of products (c) coordinate of the utopia point with applied transformation 2 on production goals 


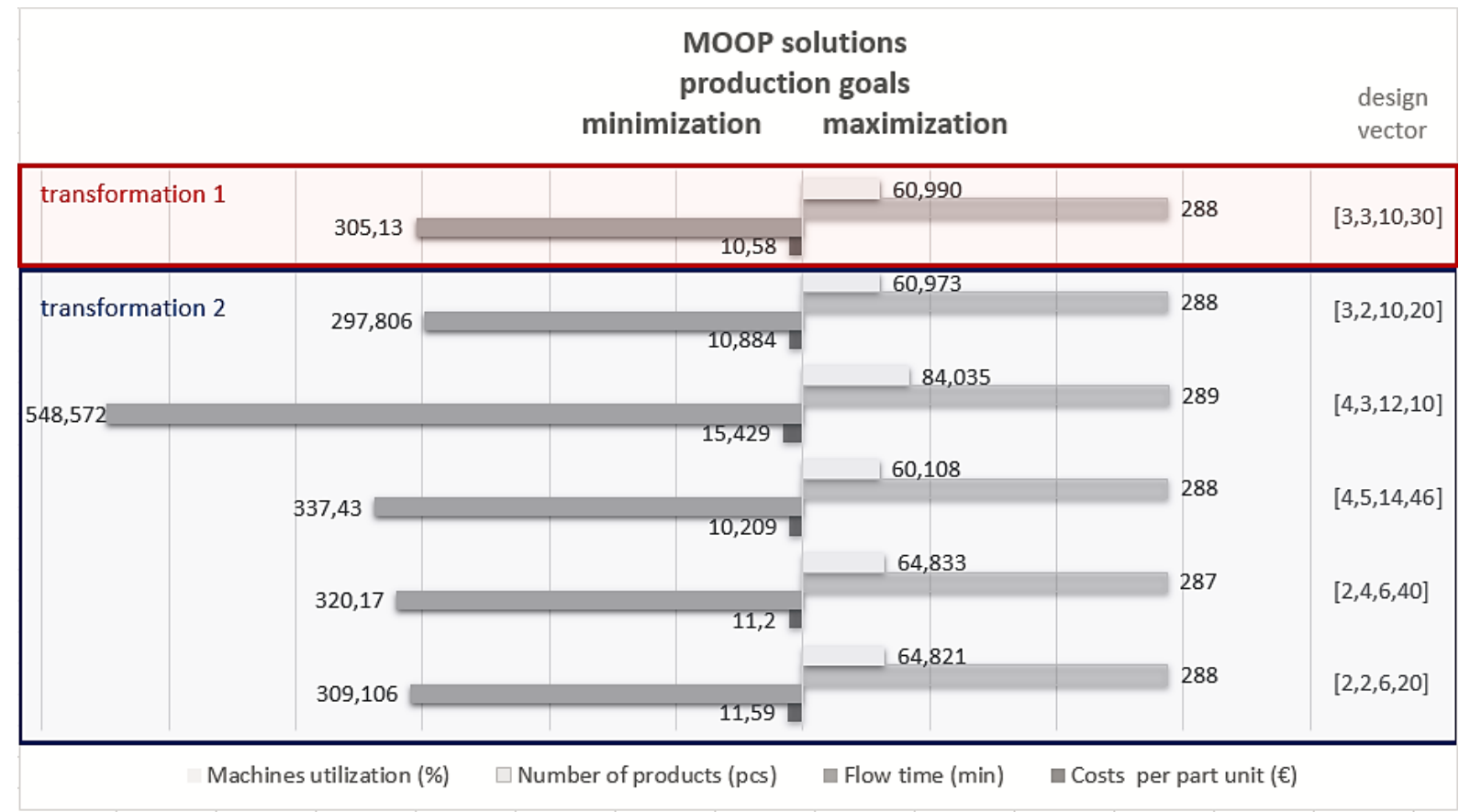

Figure 3 Solutions of MOO problem using two types of transformation. The red colour represents the result of the transformation 1 offering a compromise solution.

\section{DISCUSSION ON RESULTS}

With respect to transformation 2, Figure 2 indicates that all obtained solutions were little sensitive to changing the coordinate of number of products or flow time in the utopia point for the model production system. On the other hand, the changing the costs per part unit component in the utopia point vector influenced them significantly. We can observe that small values of this coordinate caused the growing values of all production goals.

As can be seen in Figure 3, the optimization experiment based on the applying the transformation 1 have provided one compromise solution regarding to production goals. The scalar multi-objective optimization with using the transformation 2 (the variation of the utopia point setting) found five different solutions with minimal value of scalar multi-objective function for all optimization experiments. In this case we observed very low sensitivity of the number of products to the applied transformation. On the contrary, the bar graph shows the wide range of flow time change. Additionally, with respect to the design vector values, one can say there exists such utopia point setting through transformation 2 that increases all production goals simultaneously even though it is contra productive if we wish to decrease costs per part unit or to reduce the flow time.

\section{CONCLUSION}

An applying weighted-sum method based on parametric modelling in a multi-objective simulation optimization demands the normalization of individual objectives. The parameters setting (such as weights or coordinates of a utopia point) in scalar multi-objective function is not wholly clear so the estimation by an engineering intuition is commonly used. The aim of this study was to find how the setting of a utopia point coordinates influences the optimal solutions of scalar MOO problem in the terms of production goals, and thus also corresponding design of a production system loading.

This investigation pointed out that the applied transformation in scalar multi-objective function using weighted-sum method can influence the MOO problem solutions yielded by 
simulation optimization. Predominantly, the costs per part unit coordinate demonstrated its effect most strongly. Transformation which takes into account production limits of the system for setting the utopia and nadir points offered one compromise solution for specified limits. Transformation with different setting of utopia point offered more variability, which was mainly due to the variation of costs per part unit coordinate.

\section{Acknowledgement}

This paper was supported by the Slovak Scientific Grant Agency VEGA within the Project $1 / 0232 / 18$ "Using the methods of multi-objective optimization in a production processes control".

This publication has been written thanks to support of the Operational Program Research and Innovation for the project Research, modelling and simulation of industrial production processes using progressive technologies, ITMS code: NFP313010T589 co-financed by the European Regional Development Fund and also for the project Research of advanced methods of intelligent information processing, ITMS code: NFP313010T570 co-financed by the European Regional Development Fund.

\section{References}

[1] NEGAHBAN, A., SMITH, J. S. 2014. Simulation for manufacturing system design and operation: Literature review and analysis. Journal of Manufacturing Systems 33(2), pp. 241-261. DOI 10.1016/j.jmsy.2013.12.007

[2] HUNTER, S. et al. An Introduction to Multi-Objective Simulation Optimization [Online]. [Accessed: 08-2019] Available at http://www.optimizationonline.org/DB_HTML/2017/03/5903.html.

[3] AMARAN, S., SAHINIDIS, N.V., SHARDA, B. ET AL. 2016. Simulation optimization: a review of algorithms and applications. Ann. Oper. Res.240, pp.351-380. DOI 10.1007/s10479015-2019-x

[4] MARLER, R.T., ARORA, J. S. 2004. Survey of Multi-Objective Optimization Methods for Engineering. Structural and Multidisciplinary Optimization 26(6), pp. 369-395. DOI 10.1007/s00158-003-0368-6

[5] YOSHIMURA, M. 2010. System Design Optimization for Product Manufacturing. London: Springer-Verlag, DOI 10.1007/978-1-84996-008-3

[6] MARLER, R.T., ARORA, J. S. 2005. Function-transformation methods for multi-objective optimization. Engineering Optimization, 37(6), pp. 551-570. DOI 10.1080/03052150500114289

[7] KIM, I. Y, DE WECK, O. 2006. Adaptive weighted sum method for multiobjective optimization: a new method for Pareto front generation. Struct. Multidisc. Optimization 31, pp. 105-116. DOI 10.1007/s00158-005-0557-6

[8] XIANG, Y., ARORA, J. S., RAHMATALLA, S., MARLER, T., BHATT, R., ABDEL-MALEK K. 2010. Human lifting simulation using a multi-objective optimization approach. Multibody Syst. Dyn. 23, pp. 431-451. DOI 10.1007/s11044-009-9186-y

[9] JAVIDTASH, N., JABBARI, M., NIKNAM, T., NAFAR, M. 2017. A novel mixture of nondominated sorting genetic algorithm and fuzzy method to multi-objective placement of distributed generations in Microgrids. Journal of Intelligent \& Fuzzy Systems 33(4), pp. 2577 2584. DOI 10.3233/JIFS-15934

\section{ORCID}

Pavel Važan

Zuzana Červeňanská

Janette Kotianová
0000-0001-6943-2635

0000-0002-6380-4634

0000-0003-2105-3155 